Objectives: We evaluated the role of these metabolic pathways in RA-FLS proliferation and in autoimmune arthritis in SKG mice.

Methods: The expression of glycolysis- or glutaminolysis-related enzymes was evaluated by real-time PCR and Western blotting, and the intracellular metabolites were evaluated by metabolomic analyses. The effects of glucose or glutamine on RA-FLS cell growth were investigated using glucose- or glutamine-free medium. Glutaminase 1 (GLS1) siRNA and the GLS1 inhibitor compound 968 were used to inhibit GLS1 in RA-FLS. Arthritis was induced in SKG mice by zymosan A injection. Compound 968 was used to study the effect of GLS1 inhibition on Zymosan A-injected SKG mice. Ki-67-positive cells were analyzed by immunohistochemistry.

Results: GLS1 expression was increased in RA-FLS, and metabolomic analyses revealed that glutamine and glutamate consumption were increased in RA-FLS. RA-FLS proliferation was reduced under glutamine-deprived, but not glucosedeprived conditions. Cell growth of RA-FLS was inhibited by GLS1 siRNA transfection or GLS1 inhibitor treatment. Silencing of GLS1 in RA-FLS did not affect IL-6 or MMP-3 production in supernatants. GLS1 expression in RA-FLS was not affected by pro-inflammatory cytokine stimulation. Compound 968 ameliorated the autoimmune arthritis and decreased the number of Ki-67-positive synovial cells in SKG mice.

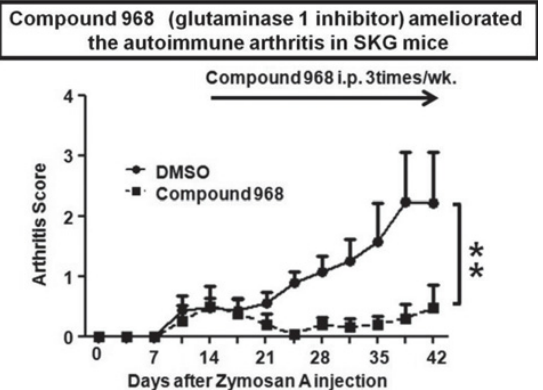

Conclusions: Our findings suggested that glutamine metabolism plays an important role in regulating RA-FLS proliferation, without being affected by proinflammatory cytokine stimulation or affecting cytokine production, and may be a novel therapeutic target for RA.

Disclosure of Interest: None declared

DOI: 10.1136/annrheumdis-2017-eular.2804

\section{FRI0023 ARTESUNATE CAN INHIBIT MIGRATION AND INVASION OF FIBROBLAST-LIKE SYNOVIOCYTES VIA SUPPRESSION OF MATRIX METALLOPROTEINASE 9 IN RHEUMATOID ARTHRITIS PATIENTS}

J. Jing ${ }^{1}$, T. Yan ${ }^{2}$, J.-D. Ma ${ }^{1}$, Y.-Q. Mo ${ }^{1}$, L. Dai ${ }^{1} .{ }^{1}$ Rheumatology, Sun Yat-sen Memorial Hospital, Sun Yat-sen University; ${ }^{2}$ Zhongshan School of Medicine, Sun Yat-Sen University, Guangzhou, China

Background: Evidences show that antimalarial agents of artemisinin and its derivatives such as artesunate may inhibit proinflammatory cytokines secretion from human rheumatoid arthritis (RA) fibroblast-like synoviocytes (FLS) in vitro. It has also been demonstrated that artesunate may ameliorate the symptoms of arthritis and prevent joint damage in collagen induced arthritis rat, which suggests that artesunate may be used for RA treatment. Recent studies show that RA-FLS is critical for joint destruction in RA because it can migrate and attach to cartilage and bone, and then invade them by secreting proteases such as matrix metalloproteinases (MMP) 9 in RA. However, effects of artesunate on migration and invasion of RA-FLS are poorly understood.

Objectives: To investigated the effects of artesunate on migration and invasion of RA-FLS and its underlying mechanism.

Methods: Synovial tissues were obtained from active RA patients as well as osteoarthritis (OA) and noninflammatory orthopedic arthropathies (Orth.A) patients and immumohistochemical (IHC) staining were performed for MMP9 expression. FLS isolated from these patients were analyzed for MMP9 exprssion by western blot (WB) and incubated with artesunate at different concentrations $(0 \mu \mathrm{M}, 20 \mu \mathrm{M}, 40 \mu \mathrm{M}$ and $60 \mu \mathrm{M})$, methotrexate (MTX, 10nM) or hydroxychloroquine

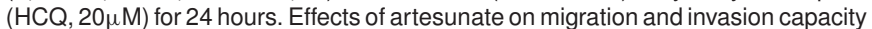
were detected by transwell and wound healing assays. MMP9 and PI3K/Akt signal transduction protein expression after artesunate treatment was measured by WB. Results: (1) IHC staining showed that synovial MMP9 expressed in lining and sublining area with intense nuclear and endochylema staining in RA synovium and the percentage of MMP9+ cells was significantly higher in RA $(n=32)$ than that in OA $(n=6)$ or Orth. A ( $n=6$, Figure $1 \mathrm{~A}, \mathrm{~B})$.

(2) Migration and wound healing assays for 12 hours and invasion assay for 24 hours showed that RA-FLS possessed stronger capacity in migration and invasion than OA-FLS or Orth.A-FLS (Figure 1E, F). Artesunate inhabits the migration and invasion capacity of RA-FLS in a dose-dependent manner. MTX also has an inhibition effect on the migration and invasion of RA-FLS, but not HCQ (Figure 2A).

(3) MMP9 expression in RA-FLS was significantly higher than that in OA-FLS or Orth.A-FLS (Figure 1C, D). $40 \mu \mathrm{M}$ or $60 \mu \mathrm{M}$ artesunate markedly inhibited the expression of MMP9 in RA-FLS by WB (Figure $2 \mathrm{~B}$ ).

(4) WB analysis showed artesunate suppressed generation of phophso-Akt in a dose-dependent manner which indicated that Akt activity (phophso-Akt/Akt) in $40 \mu \mathrm{M}$ and $60 \mu \mathrm{M}$ artesunate treatment groups were significantly lower than that in untreated group (Figure $2 \mathrm{C}$ ).

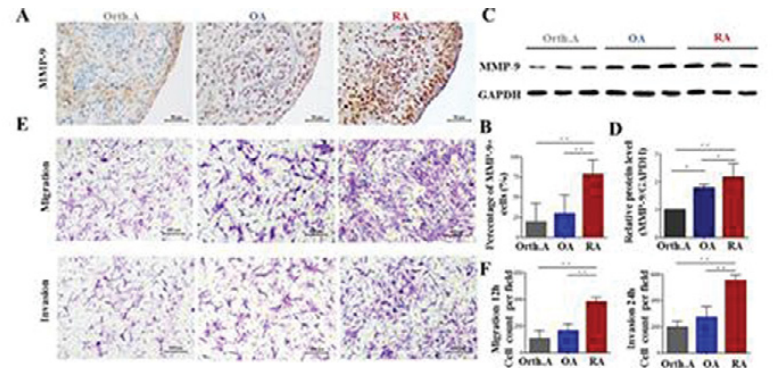

Figure 1 Expression of MMP9 in synovium and FLS and migration and invasion capacity of FLS. (A and B) IHC analysis showed that expression of MMP9 from RA patients was significantly higher than OA and Orth.A patients. Data are representative as means $\pm \mathrm{SD} * * P<0.01, * * * P<0.001$. (C and D) WB analysis showed that expression of MMP9 from RA-FLS was significantly higher than Orth.A-FLS or OA-FLS. Data were representative as means $\pm \mathrm{SD}, \bullet<<0.05, * P<0.01$. (E and $\mathbf{F}$ ) Transwell assays showed that RA-FLS possess stronger capacity of migration and invasion than OA-FLS or Orth.A-FLS. Data were representative as means $\pm \mathrm{SD}, * * P<0.01$.

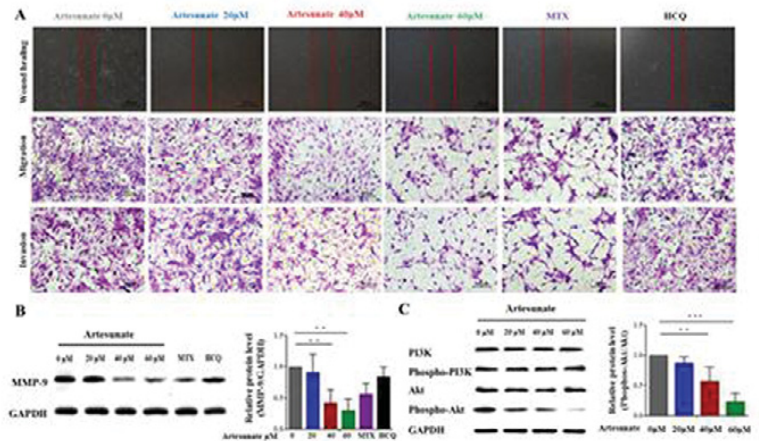

Figure 2 Effects of artesunate on the migration and invasion capacity and MMP9, PI3k/Akt pathway expression. (A) Artesunate inhabits the migration and invasion capacity of RA-FLS in a dose-dependent manner. (B) WB analysis of RA-FLS showed that MMP9 expression was siginificantly inhibited with treatment with $40 \mu \mathrm{M}$ and $60 \mu \mathrm{M}$ artesunate. (C) WB analysis showed that artesunate suppressed the expression of phophso-Akt in a dose-dependent manner. Bar are representative as means $\pm \mathrm{SD}, * P<0.01$, $\cdots p<0.001$

Conclusions: Artesunate could inhibit the migration and invasion capacity of RA-FLS and the expression of MMP9 through suppressing Akt activity.

Acknowledgements: This work was supported by National Natural Science Foundation of China (81671612 and 81471597), Research Project of Traditional Chinese Medicine Bureau of Guangdong Province (20161058) and Guangdong Natural Science Foundation (2014A030313074).

Disclosure of Interest: None declared

DOI: 10.1136/annrheumdis-2017-eular.2039

\section{FRI0024 MICRORNA-146A CONTROLS LOCAL BONE DESTRUCTION BY REGULATING FIBROBLAST INDUCED OSTEOCLASTOGENESIS IN INFLAMMATORY ARTHRITIS}

V. Saferding ${ }^{1}$, A. Puchner ${ }^{1}$, E. Goncalves-Alves ${ }^{1}$, M. Hofmann ${ }^{1}$, M. Bonelli ${ }^{1}$, S. Hayer ${ }^{1}$, H.P. Kiener ${ }^{1}$, P. Georgel ${ }^{2}$, M.I. Koenders ${ }^{3}$, G. Steiner ${ }^{1}$,

J.S. Smolen ${ }^{1}$, K. Redlich ${ }^{1}$, S. Blueml ${ }^{1}$. ${ }^{1}$ Medical University of Vienna, Vienna, Austria; ${ }^{2}$ Université de Strasbourg, Strasbourg, France; ${ }^{3}$ Radboud University Nijmegen, Nijmegen, Netherlands

Background: MicroRNA (MiR-) 146a plays an important role in the regulation of the innate immune response and has also been shown to suppress cancer development in myeloid cells. Although in late stages of arthritis elevated expression of miR-146a in synovial tissue of rheumatoid arthritis patients was detected, the level of this miRNA was found to be down regulated in early disease, but its role in the development of inflammatory arthritis is still elusive.

Objectives: The objective of this study is to analyse the role of miR-146a in arthritis by the use of a chronic arthritis disease model. We aim to investigate the regulatory function of this miRNA in the pathogenic stroma, therefore in fibroblasts but also in immune cells.

Methods: To induce arthritis we used the chronic inflammatory hTNFtg disease model, therefore we crossed miR-146a deficient into hTNFtg mice. Disease severity was assessed clinically and histologically. Blood of arthritis animals was analysed by flow cytometry. Serum cytokine levels were measured by Elisa. Synovial fibroblasts were isolated from metatarsal bones and their proliferation 
was analysed histologically and by ${ }^{3}[\mathrm{H}]$ thymidine incorporation. RNA expression levels were measured by QPCR.

Results: When we crossed miR-146a ${ }^{-/}$into hTNFtg mice, histological examination revealed a significant increase in synovial inflammation and even more striking a more than twofold increase in local bone destruction, due to increased generation of osteoclasts in the tarsal joints of miR-146a $\mathrm{a}^{-1} / \mathrm{hTNFtg}$ mice compared to hTNFtg mice. Interestingly, systemic bone loss was comparable in hTNFtg compared to miR-146a ${ }^{-1 /} / \mathrm{hTNF}$ tg mice, suggesting an important local role of miR-146a. Indeed, we detected increased levels of IL-1 $\beta$, TRAF6, a major target of miR-146a and RANKL, in addition the expression level of OPG was decreased locally in the paws of miR-146- $\mathrm{a}^{-1 /} / \mathrm{hTNFtg}$ compared to hTNFtg mice. By performing bone marrow transplants we could indeed show a pivotal role for miR-146a in mesenchymal cells in controlling local osteoclast generation and bone destruction. Analysis of important mesenchymal cells in arthritis, the synovial fibroblasts exhibited enhanced proliferation if miR-146a is missing, in vitro and in vivo. Moreover stimulation of these cells with IL-1 $\beta$, a prominent cytokine in arthritis which was also shown to be negatively regulated by miR-146a, led to increased expression of RANKL and TRAF6 in miR-146a deficient synovial fibroblasts.

Conclusions: These data demonstrate an important mitigating role of the miR$146 a$ in inflammatory arthritis, most importantly in local bone destruction, by controlling mesenchymal expression of osteoclastogenic factors. This shows an important anti-inflammatory role of miR-146a, which might possibly be exploited for therapeutic purposes.

Disclosure of Interest: None declared

DOI: 10.1136/annrheumdis-2017-eular.3384

\section{FRI0025 THE TAM RECEPTORS AXL AND MER PLAY A PROTECTIVE ROLE IN A TEMPORAL AND SPATIAL MANNER IN INFLAMMATORY ARTHRITIS}

C.E.J. Waterborg ${ }^{1}$, P.G. Través ${ }^{2}$, S. Beermann ${ }^{1}$, M.I. Koenders ${ }^{1}$, P.L.E.M. van Lent ${ }^{1}$, F. den Hoogen ${ }^{1}$, P.M. van der Kraan ${ }^{1}$, F.A.J. van de Loo ${ }^{1}$. ${ }^{1}$ Experimental Rheumatology, Radboudumc, Nijmegen, Netherlands; ${ }^{2}$ Molecular Neurobiology Laboratory, The Salk Institute for Biological Studies, la Jolla, CA, United States

Background: Rheumatoid arthritis (RA) is a chronic autoimmune disease characterized by an inflammatory response in synovial joints. One family of tyrosine kinase receptors that mediates a natural anti-inflammatory feedback mechanism are Tyro3, Axl and Mer (gene Mertk; TAM). The two principal TAM receptor protein ligands are Growth Arrest-Specific 6 (Gas6) and Protein $S$ (Pros1). Targeting the TAM receptors by adenoviral overexpression of their ligands alleviates experimental arthritis pathology. Notably, Gas6 is a ligand for all three receptors but with the highest affinity for AxI. In contrast, Pros1 can only activate Tyro3 and Mer but not Axl. The role of the separate TAM receptors in RA has not been elucidated.

Objectives: To reveal the individual role of the TAM receptors Axl and Mer in an experimental inflammatory model of RA.

Methods: The KRN serum transfer model of arthritis was induced by two

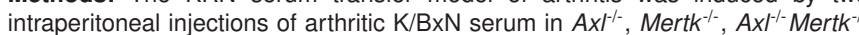
and wild-type (WT) mice. Ankle joints were macroscopically scored for 7 days. At day 0,2 and 7, ankle and knee joints were isolated for histology and immunohistochemistry.

Results: The course of arthritis was studied macroscopically and Mertk ${ }^{-1}$ mice had increased macroscopic ankle scores until day 4, compared to WT. Thereafter, $A x^{-1-}$ mice developed more severe arthritis as compared to WT with enhanced macroscopic scores from day 4 until day 7 . Histology of ankle joints showed significantly more inflammation in Mertk'- mice at day 2 and increased arthritis pathology in $\mathrm{Ax}^{-1-}$ mice at day 7 , reflecting the macroscopic ankle scores. Histological analysis of ankle joints of $A x F^{-1-}$ Mertk $^{-1-}$ mice at day 7 showed enhanced pathology compared to both $A x^{H^{-1}}$ and WT mice, indicating an additive effect of Axl and Mer deficiency. In contrast to the ankle joints at day 7, enhanced macroscopic scores and arthritis pathology in knee joints of Mertk ${ }^{-1}$ mice, compared to WT mice, was observed at both day 2 and day 7 . Axt- mice had comparable knee joint pathology compared to WT. To explain the discrepancy of Axl involvement between ankle and knee at day 7 , we examined Axl expression in synovium before the onset of arthritis. The cells in the lining layer of ankle synovium were strikingly Axl positive whereas the synovium of the knee joints was Axl negative.

Conclusions: The Mer receptor plays a protective role at the onset of arthritis whereas the Axl receptor takes over this role in established disease in ankle joints. In the knee joints, however, Mer but not Axl, plays a prominent protective role, likely due to the lack of $A x l$ in naïve knee joints. These findings identify the TAM receptors Axl and Mer as protective in arthritis, dependent on the distinct anatomic location. It is tempting to speculate that these differences in topographically distinct synovial joints could, at least in part, explain the differential joint involvement in RA.

Disclosure of Interest: None declared

DOI: 10.1136/annrheumdis-2017-eular.4887

\section{FRI0026 IDENTIFICATION OF MICRORNAS CANDIDATES SPECIFICALLY EXPRESSED IN MONOCYTES OF UNDIFFERENTIATED ARTHRITIS PATIENTS WHO PROGRESSED TO RHEUMATOID ARTHRITIS}

W. Kurowska ${ }^{1}$, E. Kuca-Warnawin ${ }^{1}$, A. Radzikowska ${ }^{1}$, M. Ciechomska ${ }^{1}$, U. Skalska ${ }^{1}$, M. Jakubaszek ${ }^{2}$, B. Kwiatkowska ${ }^{2}$, W. Maslinski ${ }^{1} .{ }^{1}$ Department of Pathophysiology and Immunology; ${ }^{2}$ Early Arthritis Clinic, National Institute of Geriatrics, Rheumatology and Rehabilitation, Warsaw, Poland

Background: Monocytes are central to the initiation of inflammation and bone erosion in rheumatoid arthritis (RA). Therefore, understanding of molecular pathways regulation in monocytes, especially at the early stages of RA development, is of crucial importance as it may help to predict the progression to the full-blown disease. However, mechanisms of gene regulation in monocytes, particularly the pattern of microRNA molecules (miRNAs) expression which can be involved in regulation of several cellular inflammatory pathways, in early phases of RA (i.e. at undifferentiated arthritis-UA stage) are not fully characterized.

Objectives: To investigate the pattern of miRNAs expression in monocytes that could serve as new biomarkers for RA development.

Methods: Magnetically sorted monocytes from peripheral blood of 19 patients with UA served for total RNA isolation. Total RNA from both sample and reference was labelled with $\mathrm{Hy} 3$ and $\mathrm{Hy} 5$ fluorescent label, respectively, using the miRCURY LNA microRNA Hi-Power Labeling Kit (Exiqon, Denmark). Fluorescent labelled samples and a reference RNA sample were hybridized to the miRCURY LNA microRNA Array 7th Gen (Exiqon), followed by scanning by Microarray Scanner System (Agilent Technologies, USA). The image analysis was carried out using ImaGene 9 Software (Exiqon).

Results: Out of 19 patients with UA enrolled in the study, 12 were verified for diagnosis after 4 years of follow-up ( 7 patients did not respond to the call for re-evaluation). Four patients developed full-blown RA (UA $\rightarrow$ RA patients), 6 patients remained still in UA phase (UA $\rightarrow$ UA patients), 1 patient was diagnosed as having Sjögren's Syndrome, and 1 undifferentiated connective tissue disease. Baseline characteristics of UA $\rightarrow R A$ vs UA $\rightarrow$ UA patients were as follows: age (median: 50.5, range 37-59 years vs 52.5, range 32-63 years), CRP (median: 9.0, range $1-22 \mathrm{mg} / \mathrm{l}$ vs 8.0 , range $1-21 \mathrm{mg} / \mathrm{l}$ ), ESR (median: 37.0 , range $4-47 \mathrm{~mm} / \mathrm{h}$ vs 34.0, range $15-44 \mathrm{~mm} / \mathrm{h}$ ) and swollen joins count (median: 3.0, range 1-9 vs 2.5 , range 1-4). Following computational unsupervised analysis we identified 50 miRNAs in monocytes that have the largest variation across all patients samples. From these 50 miRNAs we selected several specific miRNA candidates on the basis of significantly changed expression in monocytes of UA $\rightarrow R A$ vs $\cup A \rightarrow U A$ patients. Predicted specific miRNAs targeting inflammatory genes in monocytes of UA $\rightarrow$ RA patients are: miR-483-3p (2.7-fold increased, $p=0.009$ ), miR-378d (4.1-fold decreased, $p=0.0059$ ), miR-371b-5p (52.8-fold increased, $\mathrm{p}=0.0381$ ) miR-642b-5p (13.7-fold increased, $\mathrm{p}=0.0380$ ), and miR-25-3p (1.8-fold decreased, $p=0.0317$ ). Additional validation of selected miRNAs candidates will be further performed using QPCR analysis.

Conclusions: Our results indicate new miRNA candidates differentially expressed in peripheral blood monocytes from patients with UA who subsequently developed RA, in comparison to patients with UA who did not progress to RA after 4 years follow-up.

Acknowledgements: Supported by NCN Poland (grant 2012/06/A/NZ5/00059). Disclosure of Interest: None declared

DOI: 10.1136/annrheumdis-2017-eular.5703

\section{FRI0027 TNF EXPRESSION ON MICROPARTICLES FROM RHEUMATOID ARTHRITIS PATIENTS MEDIATES ENDOTHELIAL CELL FATE IN VITRO}

C. Barbati, M. Vomero, T. Colasanti, L. Novelli, F.R. Spinelli, F. Miranda,

F. Ceccarelli, A. Finucci, C. Perricone, R. Scrivo, F. Conti, G. Valesini,

C. Alessandri. Department of Internal Medicine and Medical Specialties, Arthritis Center, Sapienza University of Rome, Rome, Italy, roma, Italy

Background: Microparticles (MPs) are small membrane vesicles released by many cell types under physiological conditions or pathological situations. Increased levels of MPs have been reported in patients with autoimmune diseases, such as Rheumatoid Arthritis (RA) (1) which is characterized by an accelerated atherosclerosis. TNF, a key cytokine involved in the pathogenesis of RA, has been associated to RA atherosclerosis (2). Moreover, MPs could also have a role in endothelial dysfunction contributing to atherosclerosis in RA patients.

Objectives: The aim of this study was: 1) to evaluate TNF expression on RA-MPs. 2) to estimate the effects of serum RA-MPs on endothelial apoptosis and autophagy before and after in vivo and in vitro treatment with Etanercept. Methods: 15 RA patients were recruited from the Department of Rheumatology Sapienza University of Rome at baseline (TO) and after three months of therapy (T3) with Etanercept. A fasting blood sample was collected and centrifuged two times to obtain platelet-poor plasma rich in MPs. The resulting plasma was stained with Ab anti-TNF and analized by flow cytometry. In vitro effects of serum RA-MPs on endothelium were evaluated using human umbilical vein cell line EA.hy926. Cells were treated with RA-MPs purified at T0 and T3 and with RA-MPs in vitro treated with Etanercept. At the end of experiments apoptosis and autophagy were evaluated. Apoptosis was analyzed by flow cytometry using a FITC-conjugated 